\title{
Nonlinear response of cantilever beams to combination and subcombination resonances
}

\author{
Ali H. Nayfeh * and Haider N. Arafat \\ Department of Engineering Science and Mechanics, \\ MC 0219, Virginia Polytechnic Institute and State \\ University, Blacksburg, VA 24061, USA
}

Received 11 May 1998

Revised 18 September 1998

The nonlinear planar response of cantilever metallic beams to combination parametric and external subcombination resonances is investigated, taking into account the effects of cubic geometric and inertia nonlinearities. The beams considered here are assumed to have large length-to-width aspect ratios and thin rectangular cross sections. Hence, the effects of shear deformations and rotatory inertia are neglected. For the case of combination parametric resonance, a two-mode Galerkin discretization along with Hamilton's extended principle is used to obtain two second-order nonlinear ordinary-differential equations of motion and associated boundary conditions. Then, the method of multiple scales is applied to obtain a set of four first-order nonlinear ordinarydifferential equations governing the modulation of the amplitudes and phases of the two excited modes. For the case of subcombination resonance, the method of multiple scales is applied directly to the Lagrangian and virtual-work term. Then using Hamilton's extended principle, we obtain a set of four first-order nonlinear ordinary-differential equations governing the amplitudes and phases of the two excited modes. In both cases, the modulation equations are used to generate frequency- and force-response curves. We found that the trivial solution exhibits a jump as it undergoes a subcritical pitchfork bifurcation. Similarly, the nontrivial solutions also exhibit jumps as they undergo saddle-node bifurcations.

Keywords: Beams, combination resonance, parametric resonance, subcombination resonance, bifurcations

\section{Introduction}

When a system is parametrically excited, combination parametric resonances may occur when the forc-

\footnotetext{
${ }^{*}$ Corresponding author. Tel.: +1 540231 5453; Fax: +1 540231 2290; E-mail: anayfeh@vt.edu.
}

ing frequency $\Omega \approx \omega_{i} \pm \omega_{j}$, where $\omega_{k}$ is the natural frequency of the $k$ th mode. When the excitation is direct, an external combination resonance can occur in systems with quadratic nonlinearities when $\Omega \approx \omega_{i} \pm \omega_{j}$ and in systems with cubic nonlinearities when $\Omega \approx$ $\left|\omega_{i} \pm \omega_{j} \pm \omega_{k}\right|$ or $\Omega \approx\left|2 \omega_{i} \pm \omega_{j}\right|$. An external subcombination resonance can occur when a forcing frequency is near one-half the sum or difference of two or more natural frequencies (Nayfeh and Mook [9]).

Dugundji and Mukhopadhyay [4] investigated the response of a thin cantilever metallic beam to combination parametric resonances involving the first bending and torsional modes (i.e., $\Omega \approx \omega_{B 1}+\omega_{T 1}$ ) in one case and the second bending and first torsional modes (i.e., $\Omega \approx \omega_{B 2}+\omega_{T 1}$ ) in another. Their experimental results show that the beam exhibits significant oscillations both in bending and in torsion. In addition, at large excitation amplitudes they observed the beam snapping-through and whipping around. Cartmell and Roberts [3] theoretically and experimentally investigated the stability of a cantilever beam-mass system possessing the two simultaneous combination parametric resonances $\Omega \approx \omega_{B 1}+\omega_{T 1} \approx \omega_{B 2}-\omega_{T 1}$. They analyzed their system using the method of multiple scales and found good agreement between theory and experiment within certain ranges of the excitation frequency. However, in other regions where periodic modulations can occur, the correlation was not satisfactory because the theoretical solution could not predict nonstationary responses.

Kar and Sujata [5] investigated the instability of an elastically restrained cantilever beam subjected to uniaxial and follower forces. They found that combination parametric resonances of the difference type do not occur when the force is uniaxial or supertangential, but that they are predominant when the force is tangential or subtangential. Kar and Sujata [6] also investigated the instability of a rotating, pretwisted, and preconed cantilever beam, taking into consideration the Coriolis effects. They found that the Coriolis force may increase the instability regions in the case of combination parametric resonances. 
Anderson et al. [1] experimentally investigated the response of a thin metallic cantilever beam with an initial curvature to a combination parametric excitation. The first four natural frequencies are $0.65 \mathrm{~Hz}, 5.65 \mathrm{~Hz}$, $16.19 \mathrm{~Hz}$, and $31.91 \mathrm{~Hz}$. They found that, over a range of forcing frequency above $32 \mathrm{~Hz}$, the first and fourth modes are activated by a combination parametric resonance with the first mode dominating the response.

Sridhar et al. [12] investigated the response of a hinged-clamped beam to the subcombination resonance $\Omega \approx \frac{1}{2}\left(\omega_{a} \pm \omega_{b}\right)$ and the combination resonance $\Omega \approx \omega_{a} \pm \omega_{b} \pm \omega_{c}$. Yamamoto et al. [16,17] theoretically and experimentally investigated the nonlinear response of simply-supported beams to combination and subcombination resonances, respectively. They [16] found that, in order to excite the external combination resonance, one needs a time-independent component in the excitation. However, they [17] found that the external subcombination resonance can be excited with only a harmonic excitation. In both cases, they found that only additive-type resonances can be activated. In these three studies, nonlinearities due to mid-plane stretching were included in the analysis.

The experimental results of Dugundji and Mukhopadhyay [4] and Anderson et al. [1] confirm the occurrence of such resonances in structures. More important, their results demonstrate that such resonances can be a mechanism where a high-frequency excitation can activate low-frequency large-amplitude modes. For example, the ratio of the excitation frequency to the natural frequency of lowest mode excited was approximately 18:1 in the experiments of Dugundji and Mukhopadhyay [4] and 49:1 in the experiments of Anderson et al. [1]. The analyses of Cartmell and Roberts [3] and Kar and Sujata [5,6] did not take into consideration the effect of nonlinearities inherent in the system.

In this paper, we investigate the response of a uniform thin metallic cantilever beam to either a combination parametric resonance or a subcombination resonance of two modes (see Fig. 1). Because such resonance phenomena cannot be adequately explained by using linear theories of vibrations, it is necessary to incorporate the effects of nonlinearities in the analysis. Furthermore, because the presence of a low-frequency component in the response may cause the beam to oscillate with large amplitudes, we account for both geometric and inertia nonlinearities. The method of multiple scales is used to determine two sets of four firstorder nonlinear ordinary-differential equations governing the modulation of the amplitudes and phases of the (a)

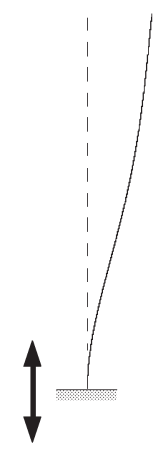

$F \cos (\Omega t)$

$\Omega \approx \omega_{i}+\omega_{j}$ (b)

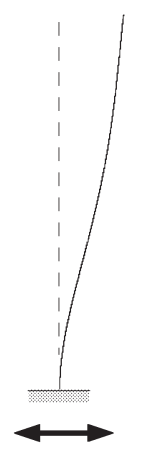

$F \cos (\Omega t)$

$\Omega \approx \frac{1}{2}\left(\omega_{i}+\omega_{j}\right)$
Fig. 1. A schematic of a cantilever beam under (a) combination parametric resonance and (b) external subcombination resonance.

two interacting modes. The modulation equations are then used to generate frequency- and force-response curves.

\section{Combination parametric resonance}

The nondimensional equation of motion for inextensional cantilever beams where the effects of shear deformation and rotatory inertia are neglected is given by

$$
\begin{aligned}
\ddot{v}+ & c \dot{v}+v^{\mathrm{iv}}=-\left(v^{\prime 2} v^{\prime \prime \prime}+v^{\prime} v^{\prime \prime 2}\right)^{\prime} \\
& -\frac{1}{2}\left(v^{\prime} \frac{\partial^{2}}{\partial t^{2}} \int_{1}^{s} \int_{0}^{s} v^{\prime 2} \mathrm{~d} s \mathrm{~d} s\right)^{\prime} \\
& +F(s, v) \cos (\Omega t),
\end{aligned}
$$

where the dimensional time $t^{*}=t \sqrt{m L^{4} / E I}$ and the dimensional deflection and arc-length are $v^{*}=L v$ and $s^{*}=L s$. The boundary conditions are

$$
\begin{array}{rlll}
v=0 & \text { and } & v^{\prime}=0 \quad \text { at } s=0, \\
v^{\prime \prime}=0 & \text { and } & v^{\prime \prime \prime}=0 & \text { at } s=1 .
\end{array}
$$

The corresponding nondimensional Lagrangian and virtual work are given by

$$
\begin{aligned}
\mathcal{L}= & \int_{0}^{1}\left\{\frac{1}{2} \dot{v}^{2}+\frac{1}{2}\left(\frac{1}{2} \frac{\partial}{\partial t} \int_{0}^{s} v^{\prime 2} \mathrm{~d} s\right)^{2}\right. \\
& \left.-\frac{1}{2}\left(v^{\prime \prime 2}+v^{\prime 2} v^{\prime \prime 2}\right)\right\} \mathrm{d} s \\
\delta W= & \int_{0}^{1} Q_{v} \delta v \mathrm{~d} s
\end{aligned}
$$




$$
=\int_{0}^{1}\{F(s, v) \cos (\Omega t)-c \dot{v}\} \delta v \mathrm{~d} s
$$

where the prime denotes differentiation with respect to the arclength $s$ and the dot denotes differentiation with respect to time $t$. Eqs (1)-(5) are valid for beams that are uniform, homogeneous, long, and thin. For stubby or thick beams, shear deformation and rotatory inertia effects may not be negligible (Timoshenko [14]).

In the presence of damping, all of the modes that are not directly excited or indirectly excited by an internal resonance will decay with time. Hence, for the case of combination parametric resonance or external subcombination resonance of the $m$ th and $n$th modes, where the $\phi_{i}$ are the orthonormal mode shapes, the long-time response of the beam will consist only of these two modes if neither of them is involved in an internal resonance with any other mode. Therefore, we assume a solution for $v$ in the form

$$
v(s, t)=\phi_{m}(s) \eta_{m}(t)+\phi_{n}(s) \eta_{n}(t) .
$$

For cantilever beams,

$$
\begin{aligned}
& \phi_{i}(s)=c_{i}\left\{\cosh \left(z_{i} s\right)-\cos \left(z_{i} s\right)\right. \\
& \left.+\frac{\cos \left(z_{i}\right)+\cosh \left(z_{i}\right)}{\sin \left(z_{i}\right)+\sinh \left(z_{i}\right)}\left[\sin \left(z_{i} s\right)-\sinh \left(z_{i} s\right)\right]\right\},
\end{aligned}
$$

where $z_{i}$ is the $i$ th root of $1+\cos (z) \cosh (z)=0$ and $c_{i}$ is chosen so that $\int_{0}^{1} \phi_{i}^{2} \mathrm{~d} s=1$. The nondimensional natural frequencies are given by

$$
\omega_{i}=z_{i}^{2}
$$

The first four nondimensional frequencies are $\omega_{1}=$ 3.5160, $\omega_{2}=22.0345, \omega_{3}=61.6972$, and $\omega_{4}=$ 120.9019

For the case of combination parametric resonance, we let

$$
F(s, v)=-\left[v^{\prime \prime}(s-1)+v^{\prime}\right] f .
$$

Substituting Eqs (6)-(9) into Eqs (4) and (5) and integrating the result over space, we obtain the discretized Lagrangian and virtual work as

$$
\mathcal{L}=\frac{1}{2}\left(1+\delta_{1} \eta_{m}^{2}+2 \delta_{2} \eta_{m} \eta_{n}+\delta_{3} \eta_{n}^{2}\right) \dot{\eta}_{m}^{2}
$$

$$
\begin{aligned}
& +\frac{1}{2}\left(1+\delta_{4} \eta_{m}^{2}+2 \delta_{5} \eta_{m} \eta_{n}+\delta_{6} \eta_{n}^{2}\right) \dot{\eta}_{n}^{2} \\
& +\left(\delta_{7} \eta_{m}^{2}+\delta_{8} \eta_{m} \eta_{n}+\delta_{9} \eta_{n}^{2}\right) \dot{\eta}_{m} \dot{\eta}_{n} \\
& -\frac{1}{2}\left(\omega_{m}^{2} \eta_{m}^{2}+\omega_{n}^{2} \eta_{n}^{2}\right)-\alpha_{1} \eta_{m}^{4}-\alpha_{2} \eta_{m}^{3} \eta_{n} \\
& -\alpha_{3} \eta_{m}^{2} \eta_{n}^{2}-\alpha_{4} \eta_{m} \eta_{n}^{3}-\alpha_{5} \eta_{n}^{4}
\end{aligned}
$$

$$
\begin{aligned}
\delta W= & -\left[2 \mu_{m} \dot{\eta}_{m}+\left(f_{m m} \eta_{m}+f_{m n} \eta_{n}\right) \cos (\Omega t)\right] \delta \eta_{m} \\
& -\left[2 \mu_{n} \dot{\eta}_{n}+\left(f_{n m} \eta_{m}+f_{n n} \eta_{n}\right) \cos (\Omega t)\right] \delta \eta_{n} \\
= & Q_{m} \delta \eta_{m}+Q_{n} \delta \eta_{n},
\end{aligned}
$$

where the $\delta_{i}, \alpha_{i}, \mu_{i}$, and $f_{i j}$ are defined in Appendix A.

By applying Hamilton's extended principle,

$$
\begin{aligned}
\frac{\mathrm{d}}{\mathrm{d} t}\left(\frac{\partial \mathcal{L}}{\partial \dot{\eta}_{m}}\right)-\frac{\partial \mathcal{L}}{\partial \eta_{m}} & =Q_{m}, \\
\frac{\mathrm{d}}{\mathrm{d} t}\left(\frac{\partial \mathcal{L}}{\partial \dot{\eta}_{n}}\right)-\frac{\partial \mathcal{L}}{\partial \eta_{n}} & =Q_{n},
\end{aligned}
$$

we obtain

$$
\begin{aligned}
\ddot{\eta}_{m}+ & 2 \mu_{m} \dot{\eta}_{m}+\omega_{m}^{2} \eta_{m} \\
= & -\left(4 \alpha_{1} \eta_{m}^{3}+3 \alpha_{2} \eta_{m}^{2} \eta_{n}+2 \alpha_{3} \eta_{m} \eta_{n}^{2}+\alpha_{4} \eta_{n}^{3}\right) \\
& -\left(\delta_{1} \eta_{m}^{2}+2 \delta_{2} \eta_{m} \eta_{n}+\delta_{3} \eta_{n}^{2}\right) \ddot{\eta}_{m} \\
& -\left(\delta_{7} \eta_{m}^{2}+\delta_{8} \eta_{m} \eta_{n}+\delta_{9} \eta_{n}^{2}\right) \ddot{\eta}_{n} \\
& -\left(\delta_{1} \eta_{m}+\delta_{2} \eta_{n}\right) \dot{\eta}_{m}^{2}-2\left(\delta_{2} \eta_{m}+\delta_{3} \eta_{n}\right) \dot{\eta}_{m} \dot{\eta}_{n} \\
& -\left[\left(\delta_{8}-\delta_{4}\right) \eta_{m}+\left(2 \delta_{9}-\delta_{5}\right) \eta_{n}\right] \dot{\eta}_{n}^{2} \\
& -\left(f_{m m} \eta_{m}+f_{m n} \eta_{n}\right) \cos (\Omega t),
\end{aligned}
$$

$$
\begin{aligned}
\ddot{\eta}_{n}+ & 2 \mu_{n} \dot{\eta}_{n}+\omega_{n}^{2} \eta_{n} \\
= & -\left(\alpha_{2} \eta_{m}^{3}+2 \alpha_{3} \eta_{m}^{2} \eta_{n}+3 \alpha_{4} \eta_{m} \eta_{n}^{2}+4 \alpha_{5} \eta_{n}^{3}\right) \\
& -\left(\delta_{7} \eta_{m}^{2}+\delta_{8} \eta_{m} \eta_{n}+\delta_{9} \eta_{n}^{2}\right) \ddot{\eta}_{m} \\
& -\left(\delta_{4} \eta_{m}^{2}+2 \delta_{5} \eta_{m} \eta_{n}+\delta_{6} \eta_{n}^{2}\right) \ddot{\eta}_{n} \\
& -\left[\left(2 \delta_{7}-\delta_{2}\right) \eta_{m}+\left(\delta_{8}-\delta_{3}\right) \eta_{n}\right] \dot{\eta}_{m}^{2} \\
& -2\left(\delta_{4} \eta_{m}+\delta_{5} \eta_{n}\right) \dot{\eta}_{m} \dot{\eta}_{n} \\
& -\left(\delta_{5} \eta_{m}+\delta_{6} \eta_{n}\right) \dot{\eta}_{n}^{2} \\
& -\left(f_{n m} \eta_{m}+f_{n n} \eta_{n}\right) \cos (\Omega t) .
\end{aligned}
$$

To determine a second-order uniform expansion for the solutions of Eqs (14) and (15) for the case of com- 
bination parametric resonance of the additive type, we scale $\mu_{i}$ and $f_{i j}$ as $\varepsilon^{2} \mu_{i}$ and $\varepsilon^{2} f_{i j}$ and introduce the detuning parameter $\sigma$ so that

$$
\Omega=\omega_{m}+\omega_{n}+\varepsilon^{2} \sigma
$$

where $\varepsilon$ is a small nondimensional bookkeeping parameter. Next, using the method of multiple scales (Nayfeh [7]), we obtain

$$
\begin{aligned}
& \eta_{m}=\varepsilon\left[A_{m}\left(T_{2}\right) \mathrm{e}^{\mathrm{i} \omega_{m} T_{0}}+\bar{A}_{m}\left(T_{2}\right) \mathrm{e}^{-\mathrm{i} \omega_{m} T_{0}}\right]+\cdots, \\
& \eta_{n}=\varepsilon\left[A_{n}\left(T_{2}\right) \mathrm{e}^{\mathrm{i} \omega_{n} T_{0}}+\bar{A}_{n}\left(T_{2}\right) \mathrm{e}^{-\mathrm{i} \omega_{n} T_{0}}\right]+\cdots,
\end{aligned}
$$

where $T_{0}=t, T_{2}=\varepsilon^{2} t$, and $A_{m}$ and $A_{n}$ are governed by

$$
\begin{gathered}
-2 \mathrm{i} \omega_{m}\left(A_{m}^{\prime}+\mu_{m} A_{m}\right)=S_{m m} A_{m}^{2} \bar{A}_{m} \\
+S_{m n} A_{m} A_{n} \bar{A}_{n}+\frac{1}{2} f_{m n} \bar{A}_{n} \mathrm{e}^{\mathrm{i} \sigma T_{2}}, \\
-2 \mathrm{i} \omega_{n}\left(A_{n}^{\prime}+\mu_{n} A_{n}\right)=S_{n n} A_{n}^{2} \bar{A}_{n} \\
+S_{n m} A_{n} A_{m} \bar{A}_{m}+\frac{1}{2} f_{n m} \bar{A}_{m} \mathrm{e}^{\mathrm{i} \sigma T_{2}},
\end{gathered}
$$

the prime indicates the derivative with respect to $T_{2}$, and

$$
\begin{aligned}
S_{m m} & =12 \alpha_{1}-2 \omega_{m}^{2} \delta_{1}, \\
S_{n n} & =12 \alpha_{5}-2 \omega_{n}^{2} \delta_{6}, \\
S_{m n} & =S_{n m}=4 \alpha_{3}-2 \omega_{m}^{2} \delta_{3}-2 \omega_{n}^{2} \delta_{4} .
\end{aligned}
$$

The $S_{i j}$ and $f_{i j}$ were calculated for combination parametric resonances of the additive type for different pairs of the first four modes. The results are shown in Table 1. It follows from Table 1 that $S_{11}>0$ and $S_{22}, S_{33}$, and $S_{44}<0$. Hence, the nonlinearity is of the hardening type for the first mode and of the softening type for the higher modes.

The complex-valued modulation equations (19) and (20) can be transformed into a real-valued form by introducing the transformation

$$
A_{m}=\frac{1}{2} a_{m} \mathrm{e}^{\mathrm{i} \beta_{m}} \quad \text { and } \quad A_{n}=\frac{1}{2} a_{n} \mathrm{e}^{\mathrm{i} \beta_{n}}
$$

Substituting Eqs (22) into Eqs (19) and (20) and separating real and imaginary parts, we obtain

$$
\begin{aligned}
a_{m}^{\prime}= & -\mu_{m} a_{m}-\frac{f_{m n}}{4 \omega_{m}} a_{n} \sin \gamma, \\
a_{m} \beta_{m}^{\prime}= & \frac{S_{m m}}{8 \omega_{m}} a_{m}^{3}+\frac{S_{m n}}{8 \omega_{m}} a_{m} a_{n}^{2} \\
& +\frac{f_{m n}}{4 \omega_{m}} a_{n} \cos \gamma, \\
a_{n}^{\prime}= & -\mu_{n} a_{n}-\frac{f_{n m}}{4 \omega_{n}} a_{m} \sin \gamma, \\
a_{n} \beta_{n}^{\prime}= & \frac{S_{n m}}{8 \omega_{n}} a_{m}^{2} a_{n}+\frac{S_{n n}}{8 \omega_{n}} a_{n}^{3}+\frac{f_{n m}}{4 \omega_{n}} a_{m} \cos \gamma,
\end{aligned}
$$

where

$$
\gamma \equiv \sigma T_{2}-\beta_{m}-\beta_{n}
$$

Substituting Eqs (22) into Eqs (17) and (18) and then substituting the result into Eq. (6), we find that the beam response is given by

$$
\begin{aligned}
& v(s, t) \approx \varepsilon\left[a_{m} \phi_{m}(s) \cos \left(\omega_{m} t+\beta_{m}\right)\right. \\
& \left.+a_{n} \phi_{n}(s) \cos \left(\omega_{n} t+\beta_{n}\right)\right],
\end{aligned}
$$

where the $a_{i}$ and $\beta_{i}$ are given by Eqs (23)-(27). Using Eqs (16) and (27) to eliminate $\omega_{n}$ and $\beta_{n}$ from Eq. (28), we have

$$
\begin{aligned}
& v(s, t) \approx \varepsilon\left\{a_{m} \phi_{m}(s) \cos \left(\omega_{m} t+\beta_{m}\right)\right. \\
& \left.\quad+a_{n} \phi_{n}(s) \cos \left[\left(\Omega-\omega_{m}\right) t-\beta_{m}-\gamma\right]\right\}
\end{aligned}
$$

The equilibrium solutions or fixed points of Eqs (23)-(27) correspond to $a_{m}^{\prime}=0, a_{n}^{\prime}=0$, and $\gamma^{\prime}=$ 0 , which in turn correspond to two-period quasiperiodic responses of the beam according to Eq. (29). There are two possible equilibrium solutions: (a) $a_{m}=$ 0 and $a_{n}=0$ and the beam is not excited and (b) $a_{m} \neq$ 0 and $a_{n} \neq 0$ and the beam response is quasiperiodic. In the latter case, Eqs (24), (26), and (27) can be used to eliminate $\beta_{m}$ and $\beta_{n}$ to obtain the following equation for $\gamma$ :

$$
\begin{aligned}
\gamma^{\prime}= & \sigma-\left(\frac{S_{m m}}{8 \omega_{m}}+\frac{S_{n m}}{8 \omega_{n}}\right) a_{m}^{2}-\left(\frac{S_{m n}}{8 \omega_{m}}+\frac{S_{n n}}{8 \omega_{n}}\right) a_{n}^{2} \\
& -\left(\frac{a_{n} f_{m n}}{4 a_{m} \omega_{m}}+\frac{a_{m} f_{n m}}{4 a_{n} \omega_{n}}\right) \cos \gamma
\end{aligned}
$$

Thus, for nontrivial solutions, the modulation equations are reduced from four to three first-order differential equations. For equilibrium solutions, we set the 
Table 1

Values of the coefficients $\alpha_{i}, \delta_{i}, S_{i j}$, and $f_{i j}$ for different combinations of the first four modes

\begin{tabular}{crrr}
\hline Modes $m \& n$ & \multicolumn{1}{c}{$S_{m m}$} & \multicolumn{1}{c}{$S_{m n}=S_{n m}$} & \multicolumn{1}{c}{$S_{n n}$} \\
\hline $1 \& 2$ & 7.6680 & -1122.2408 & -100279.6731 \\
$1 \& 3$ & 7.6680 & -15928.9047 & -6818871.8310 \\
$2 \& 3$ & -100279.6731 & -75835.2975 & -6818871.8310 \\
$1 \& 4$ & 7.6680 & -34594.5220 & $-1.1042 \times 10^{8}$ \\
$2 \& 4$ & -100279.6731 & -927411.9209 & $-1.1042 \times 10^{8}$ \\
$3 \& 4$ & -6818871.8310 & -4017517.1670 & $-1.1042 \times 10^{8}$ \\
\hline Modes $m \& n$ & $f_{m m}$ & $f_{m n}=f_{n m}$ & $f_{n n}$ \\
\hline $1 \& 2$ & $1.5709 f$ & $-0.4223 f$ & $8.6471 f$ \\
$1 \& 3$ & $1.5709 f$ & $-1.0721 f$ & $24.9521 f$ \\
$2 \& 3$ & $8.6471 f$ & $1.8901 f$ & $24.9521 f$ \\
$1 \& 4$ & $1.5709 f$ & $-0.8731 f$ & $51.4591 f$ \\
$2 \& 4$ & $8.6471 f$ & $-3.6434 f$ & $51.4591 f$ \\
$3 \& 4$ & $24.9521 f$ & $8.3383 f$ & $51.4591 f$ \\
\hline
\end{tabular}

time derivatives in Eqs (23), (25), and (30) equal to zero and solve for $a_{m}, a_{n}$, and $\gamma$, yielding the following closed-form solution:

$$
\begin{aligned}
\alpha_{e} a_{m}^{2} & =\sigma \pm \frac{\mu_{m}+\mu_{n}}{\sqrt{\mu_{m} \mu_{n}}} \sqrt{\frac{f_{m n} f_{n m}}{16 \omega_{m} \omega_{n}}-\mu_{m} \mu_{n}}, \\
a_{n}^{2} & =\frac{\mu_{m} \omega_{m} f_{n m}}{\mu_{n} \omega_{n} f_{m n}} a_{m}^{2} \\
\sin \gamma & =-\frac{4 \mu_{m} \omega_{m}}{f_{m n}} \frac{a_{m}}{a_{n}}=-\frac{4 \mu_{n} \omega_{n}}{f_{n m}} \frac{a_{n}}{a_{m}} \\
& = \pm 4 \sqrt{\frac{\mu_{m} \mu_{n} \omega_{m} \omega_{n}}{f_{m n} f_{n m}}}
\end{aligned}
$$

where

$$
\begin{aligned}
\alpha_{e}= & \frac{1}{8}\left[\frac{S_{m m}}{\omega_{m}}+\frac{S_{n m}}{\omega_{n}}\right. \\
& \left.+\left(\frac{S_{m n}}{\omega_{m}}+\frac{S_{n n}}{\omega_{n}}\right) \frac{\mu_{m} \omega_{m} f_{n m}}{\mu_{n} \omega_{n} f_{m n}}\right] .
\end{aligned}
$$

The stability of a nontrivial equilibrium solution can then be studied by calculating the eigenvalues of the Jacobian matrix of Eqs (23), (25), and (30) evaluated at this equilibrium solution.

To determine the stability of the trivial equilibrium solutions, we study the stability of the linearized complex-valued modulation equations (19) and (20). To this end, we let

$$
A_{m}=c_{m} \mathrm{e}^{\lambda T_{2}+\mathrm{i} \sigma T_{2}} \quad \text { and } \quad A_{n}=c_{n} \mathrm{e}^{\bar{\lambda} T_{2}}
$$

in the linearized equations (19) and (20) and obtain

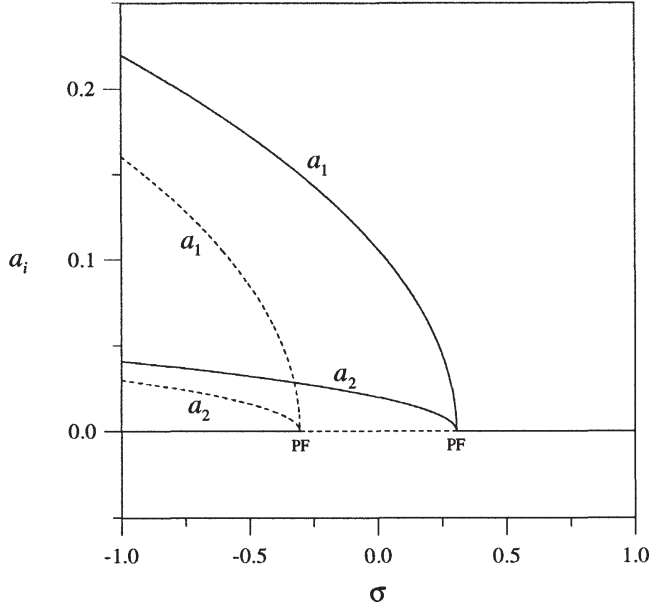

Fig. 2. Frequency-response curves for a combination parametric resonance of the additive type involving modes 1 and 2 for $f=10$, $\mu_{1}=0.0137, \mu_{2}=0.0635$. Solid lines $(-)$ denote stable fixed points and dashed lines (-- ) denote unstable fixed points.

$$
\begin{aligned}
2 \mathrm{i} \omega_{m}\left(\lambda+\mathrm{i} \sigma+\mu_{m}\right) c_{m}+\frac{1}{2} f_{m n} \bar{c}_{n} & =0, \\
2 \mathrm{i} \omega_{n}\left(\bar{\lambda}+\mu_{n}\right) c_{n}+\frac{1}{2} f_{n m} \bar{c}_{m} & =0 .
\end{aligned}
$$

Hence,

$$
\begin{aligned}
\lambda= & -\frac{1}{2}\left(\mu_{m}+\mu_{n}+\mathrm{i} \sigma\right) \pm\left(\frac{1}{4}\left(\mu_{m}+\mu_{n}+\mathrm{i} \sigma\right)^{2}\right. \\
& \left.-\mu_{n}\left(\mu_{m}+\mathrm{i} \sigma\right)+\frac{f_{m n} f_{n m}}{16 \omega_{m} \omega_{n}}\right)^{1 / 2} .
\end{aligned}
$$

It follows from Eqs (35) that the trivial solution is sta- 

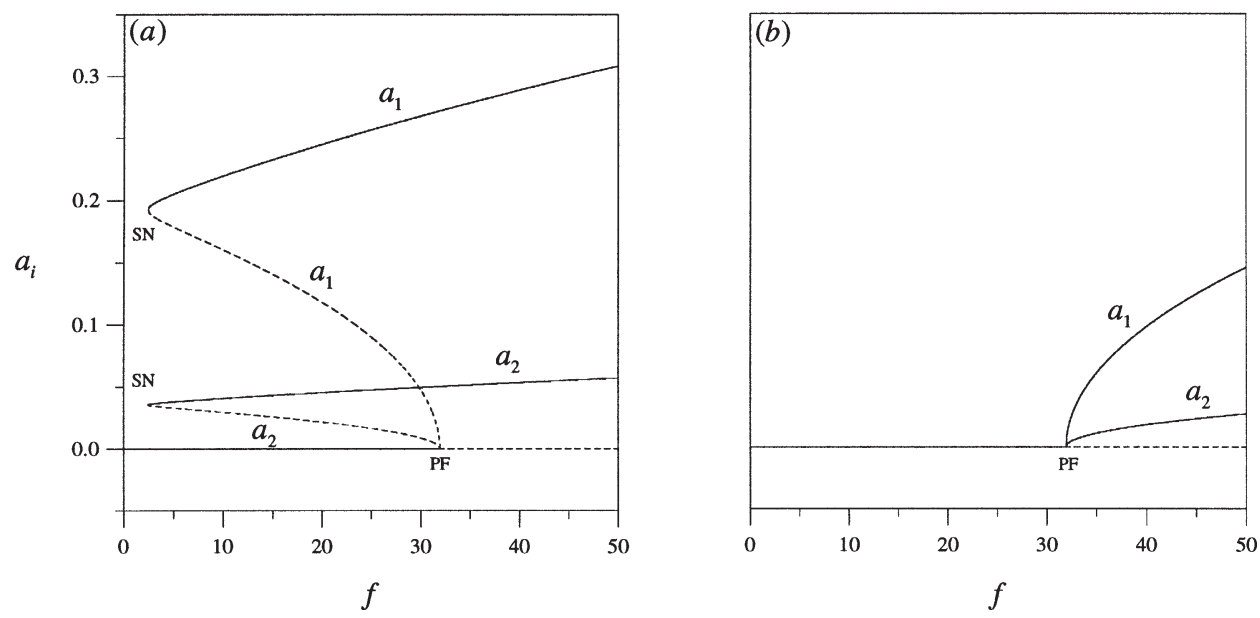

Fig. 3. Amplitude-response curves for a combination parametric resonance of the additive type involving modes 1 and 2 when $\mu_{1}=0.0137$ and $\mu_{2}=0.0635$. In part (a), $\sigma=-1$ and in part (b), $\sigma=1$. Solid lines ( - ) denote stable fixed points and dashed lines $(---)$ denote unstable fixed points.

ble if the real parts of both $\lambda$ 's are negative.

In Fig. 2, we show typical frequency-response curves for a combination parametric resonance of the additive type of the first two modes when the excitation amplitude is $f=10$. Clearly, the first mode dominates the response. Although the nonlinearity is hardening for the first mode and softening for the second mode, the frequency-response curves are bent to the left, indicating a softening behavior for both modes. This is so because

$$
\beta_{1}^{\prime}=\frac{S_{11}}{8 \omega_{1}} a_{1}^{2}+\frac{S_{12}}{8 \omega_{1}} a_{2}^{2}+\frac{f_{12} a_{2}}{4 \omega_{1} a_{1}} \cos \gamma
$$

according to Eq. (24). Although $S_{11}$ is positive, $S_{12}$ is negative and its magnitude is much larger than $S_{11}$. Hence, the nonlinearity decreases the frequency of the first mode, and hence bends the frequency-response curves to the left. It follows from Fig. 2 that, depending on how $\sigma$ is varied, the trivial solution loses stability via either a subcritical or a supercritical pitchfork bifurcation.

In Fig. 3, we show amplitude-response curves for a combination parametric resonance of the additive type of the first two modes. In part (a), the frequency detuning parameter $\sigma=-1$, and in part (b) $\sigma=1$. When $\sigma=-1$, there are two branches of nontrivial fixedpoint solutions, one stable and the other unstable. As $f$ is increased away from zero, the trivial solution loses stability via a subcritical pitchfork bifurcation, causing the response to jump up to the stable branch of nontrivial solutions. Similarly, a fixed-point on the stable nontrivial branch loses stability via a saddle-node bifurca-

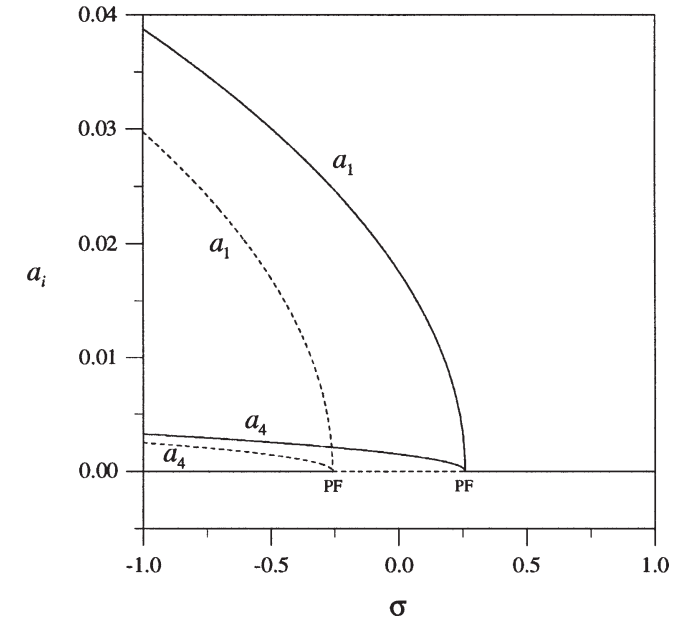

Fig. 4. Frequency-response curves for a combination parametric resonance of the additive type involving modes 1 and 4 for $f=10$, $\mu_{1}=0.0137, \mu_{4}=0.0573$. Solid lines $(-)$ denote stable fixed points and dashed lines (-- ) denote unstable fixed points.

tion as $f$ is decreased, resulting in a jump down to the trivial branch. When $\sigma \geqslant 0$, there are only branches of stable nontrivial fixed points, as shown in Fig. 3(b). The nontrivial solution is activated gradually as the trivial solution undergoes a supercritical pitchfork bifurcation.

In Figs 4 and 5, the frequency- and amplituderesponse curves are presented when the first and fourth modes are activated by the combination parametric resonance. The forcing amplitude in Fig. 4 is $f=10$ and the detuning parameter is $\sigma=-1$ in Fig. 5(a) and $\sigma=1$ in Fig. 5(b). We note that the behaviors in Figs 4 and 5 are similar to those in Figs 2 and 3. However, the 

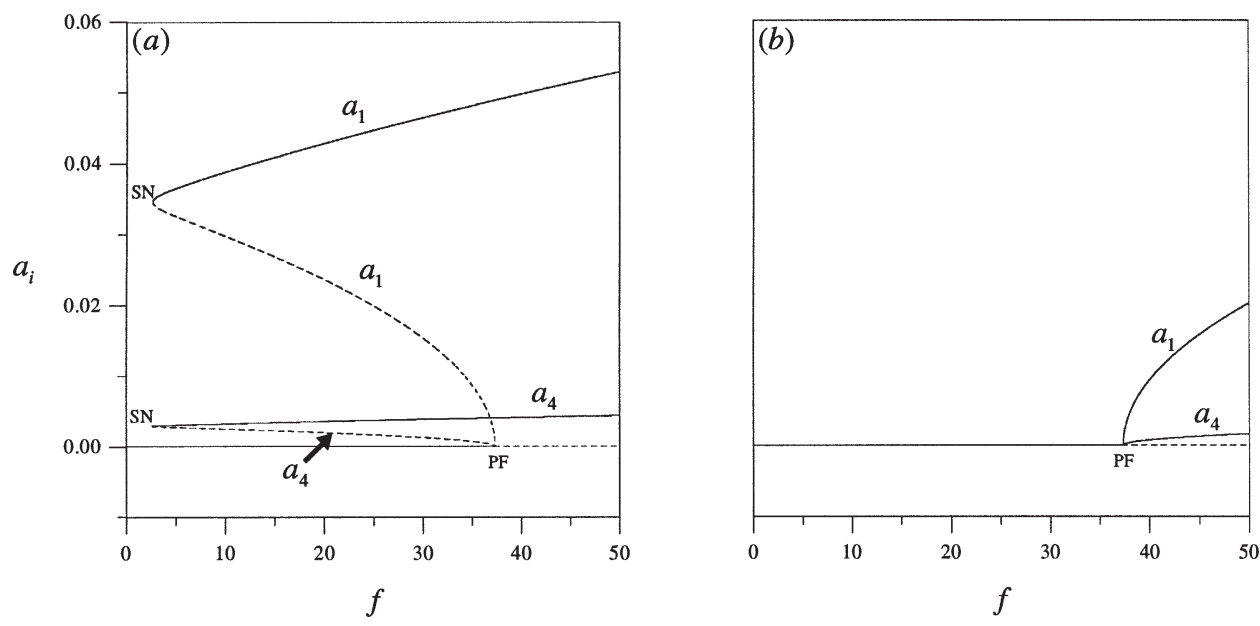

Fig. 5. Amplitude-response curves for a combination parametric resonance of the additive type involving modes 1 and 4 when $\mu_{1}=0.0137$ and $\mu_{4}=0.0573$. In part (a), $\sigma=-1$ and in part (b), $\sigma=1$. Solid lines (-) denote stable fixed points and dashed lines (- --$)$ denote unstable fixed points.

amplitudes when modes 1 and 4 are excited are about an order of magnitude smaller than those when modes 1 and 2 are excited.

Anderson et al. [1] experimentally investigated the response of a cantilever beam where $\Omega \approx 2 \omega_{3} \approx$ $\omega_{1}+\omega_{4}$. They found that over a small region of frequency detuning, only the first and fourth modes were excited by a combination parametric resonance. The results shown in Fig. 4 agree qualitatively with their frequency-response curves.

Results for the case of a combination parametric resonance of the difference type can be obtained by replacing $\omega_{m}$ by $-\omega_{m}$ and $\beta_{m}$ by $-\beta_{m}$ in Eqs (23) $-(32)$. However, it can be seen from Eq. (32) that this resonance cannot be activated in this system.

\section{External subcombination resonance}

In this section, we consider the response of the beam to the subcombination resonance $\Omega \approx \frac{1}{2}\left(\omega_{n} \pm \omega_{m}\right)$. In this case, the excitation, which is transverse, is assumed to be hard. Therefore, we let $F(s, v)=\varepsilon f(s)$ in Eqs (1) and (5). Furthermore, in order that the cubic nonlinearities and damping balance the resonance, we scale $c$ as $\varepsilon^{2} c$. We use the method of time-averaged Lagrangian and virtual work to determine a uniform first-order expansion. To this end, we let

$$
\begin{aligned}
& v\left(s, T_{0}, T_{2}\right) \approx \varepsilon\left[A_{m}\left(T_{2}\right) \phi_{m}(s) \mathrm{e}^{\mathrm{i} \omega_{m} T_{0}}\right. \\
& \left.\quad+A_{n}\left(T_{2}\right) \phi_{n}(s) \mathrm{e}^{\mathrm{i} \omega_{n} T_{0}}+\Phi(s) \mathrm{e}^{\mathrm{i} \Omega T_{0}}+c c\right],
\end{aligned}
$$

where $\phi_{m}(s)$ and $\phi_{n}(s)$ are the mode shapes corresponding to the natural frequencies $\omega_{m}$ and $\omega_{n}$ and $\Phi(s)$ is governed by the boundary-value problem

$$
\begin{aligned}
& \Phi^{i v}-\Omega^{2} \Phi=\frac{1}{2} f(s), \\
& \Phi(0)=0, \quad \Phi^{\prime}(0)=0, \\
& \Phi^{\prime \prime}(1)=0, \quad \text { and } \quad \Phi^{\prime \prime \prime}(1)=0 .
\end{aligned}
$$

We note that $2 \varepsilon \Phi(s) \cos (\Omega t)$ is the particular solution of the linear undamped beam equation and associated boundary conditions. When $f(s)$ is constant, the solution of Eqs (41) and (42) can be expressed as

$$
\begin{aligned}
\Phi(s)= & c_{1} \sin (\sqrt{\Omega} s)+c_{2} \cos (\sqrt{\Omega} s) \\
& +c_{3} \sinh (\sqrt{\Omega} s) \\
& +c_{4} \cosh (\sqrt{\Omega} s)-\frac{f}{2 \Omega^{2}}
\end{aligned}
$$

where

$$
\begin{aligned}
c_{1} & =-c_{3} \\
& =\frac{f}{4 \Omega^{2}} \frac{\sin \sqrt{\Omega} \cosh \sqrt{\Omega}+\cos \sqrt{\Omega} \sinh \sqrt{\Omega}}{1+\cos \sqrt{\Omega} \cosh \sqrt{\Omega}}, \\
c_{2} & =\frac{f}{4 \Omega^{2}} \frac{1+\cos \sqrt{\Omega} \cosh \sqrt{\Omega}-\sin \sqrt{\Omega} \sinh \sqrt{\Omega}}{1+\cos \sqrt{\Omega} \cosh \sqrt{\Omega}},
\end{aligned}
$$


$c_{4}=\frac{f}{4 \Omega^{2}} \frac{1+\cos \sqrt{\Omega} \cosh \sqrt{\Omega}+\sin \sqrt{\Omega} \sinh \sqrt{\Omega}}{1+\cos \sqrt{\Omega} \cosh \sqrt{\Omega}}$.

Clearly, Eqs (44)-(46) break down when $\Omega$ is near any of the natural frequencies of the beam. In the present case, $\Omega \approx \frac{1}{2}\left(\omega_{m} \pm \omega_{n}\right)$, which is away from any of the natural frequencies.

Next, we introduce the detuning parameter $\sigma$ such that

$$
\Omega=\frac{1}{2}\left(\omega_{m}+\omega_{n}\right)+\varepsilon^{2} \sigma .
$$

Substituting Eq. (40) into Eqs (4) and (5), using Eq. (47), and retaining only slowly varying terms, we obtain the following time-averaged Lagrangian and virtual work:

$$
\begin{aligned}
\frac{\langle\mathcal{L}\rangle}{\varepsilon^{4}}= & \mathrm{i} \omega_{m}\left(A_{m} \bar{A}_{m}^{\prime}-\bar{A}_{m} A_{m}^{\prime}\right) \\
& +\mathrm{i} \omega_{n}\left(A_{n} \bar{A}_{n}^{\prime}-\bar{A}_{n} A_{n}^{\prime}\right)-\Gamma_{m} A_{m} \bar{A}_{m} \\
& -\Gamma_{n} A_{n} \bar{A}_{n}-\frac{1}{2} S_{m m} A_{m}^{2} \bar{A}_{m}^{2} \\
& -\frac{1}{2} S_{n n} A_{n}^{2} \bar{A}_{n}^{2}-S_{m n} A_{m} \bar{A}_{m} A_{n} \bar{A}_{n} \\
& -\Lambda\left(\bar{A}_{m} \bar{A}_{n} \mathrm{e}^{2 \mathrm{i} \sigma T_{2}}+A_{m} A_{n} \mathrm{e}^{-2 \mathrm{i} \sigma T_{2}}\right) \\
& + \text { constant }+\cdots, \\
\frac{\langle\delta W\rangle}{\varepsilon^{4}}= & -2 \mathrm{i} \omega_{m} \mu_{m}\left(A_{m} \delta \bar{A}_{m}-\bar{A}_{m} \delta A_{m}\right) \\
& -2 \mathrm{i} \omega_{n} \mu_{n}\left(A_{n} \delta \bar{A}_{n}-\bar{A}_{n} \delta A_{n}\right)+\cdots,
\end{aligned}
$$

where the $S_{i j}$ are defined in Eqs (21), the $\mu_{i}$ are defined in Appendix A, and $\Gamma_{m}, \Gamma_{n}$, and $\Lambda$ are defined in Appendix B. In Table 2, we present the numerical values for the coefficients $\Gamma_{m}, \Gamma_{n}$, and $\Lambda$ for external subcombination resonances of the additive type for different pairs of the first four modes. Applying Hamilton's principle to Eqs (48) and (49), we obtain the modulation equations

$$
\begin{aligned}
& -2 \mathrm{i} \omega_{m}\left(A_{m}^{\prime}+\mu_{m} A_{m}\right)=\Gamma_{m} A_{m}+S_{m m} A_{m}^{2} \bar{A}_{m} \\
& \quad+S_{m n} A_{m} A_{n} \bar{A}_{n}+\Lambda \bar{A}_{n} \mathrm{e}^{2 \mathrm{i} \sigma T_{2}}, \\
& -2 \mathrm{i} \omega_{n}\left(A_{n}^{\prime}+\mu_{n} A_{n}\right)=\Gamma_{n} A_{n}+S_{n m} A_{m} \bar{A}_{m} A_{n} \\
& \quad+S_{n n} A_{n}^{2} \bar{A}_{n}+\Lambda \bar{A}_{m} \mathrm{e}^{2 \mathrm{i} \sigma T_{2}} .
\end{aligned}
$$

Substituting the polar transformation, Eqs (22), into Eqs (50) and (51) and separating real and imaginary
Table 2

Values of the coefficients $\Gamma_{m}, \Gamma_{n}$, and $\Lambda$ for different combinations of the first four modes

\begin{tabular}{ccrr}
\hline Modes $m \& n$ & $\Gamma_{m}$ & \multicolumn{1}{c}{$\Gamma_{n}$} & \multicolumn{1}{c}{$\Lambda$} \\
\hline $1 \& 2$ & $-0.0107 f^{2}$ & $-0.1268 f^{2}$ & $0.0130 f^{2}$ \\
$1 \& 3$ & $-0.0005 f^{2}$ & $-0.0420 f^{2}$ & $0.0051 f^{2}$ \\
$2 \& 3$ & $-0.0111 f^{2}$ & $-0.0531 f^{2}$ & $0.0006 f^{2}$ \\
$1 \& 4$ & $-0.0588 f^{2}$ & $-15.3290 f^{2}$ & $0.9893 f^{2}$ \\
$2 \& 4$ & $-0.0045 f^{2}$ & $-0.0298 f^{2}$ & $0.0161 f^{2}$ \\
$3 \& 4$ & $-0.0135 f^{2}$ & $-0.0420 f^{2}$ & $-0.0041 f^{2}$ \\
\hline
\end{tabular}

parts, we obtain the real-valued modulation equations

$$
\begin{aligned}
a_{m}^{\prime}= & -\mu_{m} a_{m}-\frac{\Lambda}{2 \omega_{m}} a_{n} \sin \gamma, \\
a_{m} \beta_{m}^{\prime}= & \frac{\Gamma_{m}}{2 \omega_{m}} a_{m}+\frac{S_{m m}}{8 \omega_{m}} a_{m}^{3} \\
& +\frac{S_{m n}}{8 \omega_{m}} a_{m} a_{n}^{2}+\frac{\Lambda}{2 \omega_{m}} a_{n} \cos \gamma, \\
a_{n}^{\prime}= & -\mu_{n} a_{n}-\frac{\Lambda}{2 \omega_{n}} a_{m} \sin \gamma, \\
a_{n} \beta_{n}^{\prime}= & \frac{\Gamma_{n}}{2 \omega_{n}} a_{n}+\frac{S_{n m}}{8 \omega_{n}} a_{m}^{2} a_{n} \\
& +\frac{S_{n n}}{8 \omega_{n}} a_{n}^{3}+\frac{\Lambda}{2 \omega_{n}} a_{m} \cos \gamma,
\end{aligned}
$$

where

$$
\gamma \equiv 2 \sigma T_{2}-\beta_{m}-\beta_{n} .
$$

We note that, except for the linear shifts $\Gamma_{i} / 2 \omega_{i}$ in the natural frequencies, Eqs (52)-(56) have the same form as Eqs (23)-(27) obtained for the case of combination parametric resonance if we put $f_{m n}=2 \Lambda$ and replace $\sigma$ with $2 \sigma$.

There are two possible solutions for Eqs (52)-(56): (a) $a_{m}=a_{n}=0$ and (b) $a_{m} \neq 0$ and $a_{n} \neq 0$. In the first case, it follows from Eq. (40) that the beam's response is given by

$$
v(s, t)=2 \varepsilon \Phi(s) \cos (\Omega t)+\cdots,
$$

which is periodic having the same period as that of the excitation. In this case, the external subcombination resonance is not activated. The stability of this trivial solution can be analyzed by investigating solutions of the linearized complex-valued modulation equations (50) and (51). To this end, we let

$A_{m}=c_{m} \mathrm{e}^{\lambda T_{2}+2 \mathrm{i} \sigma T_{2}} \quad$ and $A_{n}=c_{n} \mathrm{e}^{\bar{\lambda} T_{2}}$ 
in the linearized equations (50) and (51) and obtain

$$
\begin{array}{r}
{\left[2 \mathrm{i} \omega_{m}\left(\mu_{m}+\lambda+2 \mathrm{i} \sigma\right)+\Gamma_{m}\right] c_{m}+\Lambda \bar{c}_{n}=0,} \\
\Lambda \bar{c}_{m}+\left[2 \mathrm{i} \omega_{n}\left(\mu_{n}+\bar{\lambda}\right)+\Gamma_{n}\right] c_{n}=0 .
\end{array}
$$

For nontrivial solutions,

$$
\begin{aligned}
\lambda^{2} & +\left[\left(\mu_{m}+\mu_{n}\right)+\mathrm{i}\left(2 \sigma-\frac{\Gamma_{m}}{2 \omega_{m}}+\frac{\Gamma_{n}}{2 \omega_{n}}\right)\right] \lambda \\
& +\left[\left(\mu_{m} \mu_{n}-\frac{\sigma \Gamma_{n}}{\omega_{n}}+\frac{\Gamma_{m} \Gamma_{n}-\Lambda^{2}}{4 \omega_{m} \omega_{n}}\right)\right. \\
& \left.+\mathrm{i}\left(2 \sigma \mu_{n}-\frac{\Gamma_{m} \mu_{n}}{2 \omega_{m}}+\frac{\Gamma_{n} \mu_{m}}{2 \omega_{n}}\right)\right]=0 .
\end{aligned}
$$

It follows from Eqs (58) that the trivial solution loses stability as one of the $\lambda$ 's crosses the imaginary axis along the real axis from the left-half to the right-half of the complex plane.

For nontrivial solutions, we use Eqs (53), (55), and (56) to eliminate $\beta_{m}$ and $\beta_{n}$ and obtain

$$
\begin{aligned}
\gamma^{\prime}= & 2 \sigma-\frac{1}{2}\left(\frac{\Gamma_{m}}{\omega_{m}}+\frac{\Gamma_{n}}{\omega_{n}}\right) \\
& -\frac{1}{8}\left(\frac{S_{m m}}{\omega_{m}}+\frac{S_{n m}}{\omega_{n}}\right) a_{m}^{2} \\
& -\frac{1}{8}\left(\frac{S_{m n}}{\omega_{m}}+\frac{S_{n n}}{\omega_{n}}\right) a_{n}^{2} \\
& -\frac{\Lambda}{2}\left(\frac{a_{n}}{\omega_{m} a_{m}}+\frac{a_{m}}{\omega_{n} a_{n}}\right) \cos \gamma .
\end{aligned}
$$

The fixed points of Eqs (52), (54), and (62) correspond to $a_{m}^{\prime}=0, a_{n}^{\prime}=0$, and $\gamma^{\prime}=0$. They are given by

$$
\begin{aligned}
\alpha_{e} a_{m}^{2}= & 2 \sigma-\frac{1}{2}\left(\frac{\Gamma_{m}}{\omega_{m}}+\frac{\Gamma_{n}}{\omega_{n}}\right) \\
& \pm \frac{\mu_{m}+\mu_{n}}{\sqrt{\mu_{m} \mu_{n}}} \sqrt{\frac{\Lambda^{2}}{4 \omega_{m} \omega_{n}}-\mu_{m} \mu_{n},} \\
a_{n}^{2}= & \frac{\mu_{m} \omega_{m}}{\mu_{n} \omega_{n}} a_{m}^{2}, \\
\sin \gamma= & -\frac{2 \mu_{m} \omega_{m}}{\Lambda} \frac{a_{m}}{a_{n}}=-\frac{2 \mu_{n} \omega_{n}}{\Lambda} \frac{a_{n}}{a_{m}}, \\
\cos \gamma= & {\left[2 \sigma-\frac{1}{2}\left(\frac{\Gamma_{m}}{\omega_{m}}+\frac{\Gamma_{n}}{\omega_{n}}\right)\right.} \\
& -\frac{1}{8}\left(\frac{S_{m m}}{\omega_{m}}+\frac{S_{n m}}{\omega_{n}}\right) a_{m}^{2}
\end{aligned}
$$

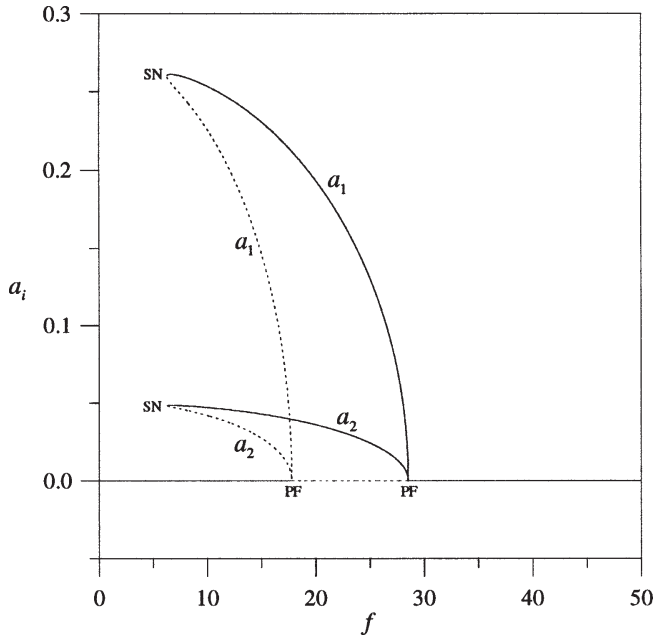

Fig. 6. Amplitude-response curves for an additive-type external subcombination resonance involving modes 1 and 2 for $\sigma=-1$, $\mu_{1}=0.0137$ and $\mu_{2}=0.0635$. Solid lines (-) denote stable fixed points and dashed lines (-- ) denote unstable fixed points.

$$
\begin{aligned}
& \left.-\frac{1}{8}\left(\frac{S_{m n}}{\omega_{m}}+\frac{S_{n n}}{\omega_{n}}\right) a_{n}^{2}\right] \\
& \times\left[\frac{\Lambda}{2}\left(\frac{a_{n}}{\omega_{m} a_{m}}+\frac{a_{m}}{\omega_{n} a_{n}}\right)\right]^{-1},
\end{aligned}
$$

where

$$
\alpha_{e}=\frac{1}{8}\left[\frac{S_{m m}}{\omega_{m}}+\frac{S_{n m}}{\omega_{n}}+\left(\frac{S_{m n}}{\omega_{m}}+\frac{S_{n n}}{\omega_{n}}\right) \frac{\mu_{m} \omega_{m}}{\mu_{n} \omega_{n}}\right] .
$$

In Fig. 6, we show typical amplitude-response curves for the subcombination external resonance of the first two modes for $\sigma=-1$. The trivial solution loses stability via a subcritical pitchfork bifurcation as the forcing amplitude is increased, resulting in a jump in the response amplitudes. On the other hand, as the forcing amplitude is decreased from a large value, the trivial solution loses stability through a supercritical pitchfork bifurcation, resulting in a gradual increase in the response amplitudes. In either case, the nontrivial solution loses stability as $f$ is decreased via a saddlenode bifurcation. Comparing Figs 3 and 6, we conclude that the linear shift in the natural frequencies $\Gamma_{i} / 2 \omega_{i}$ and the nonlinear dependence of the effective forcing $\Lambda\left(\Lambda \propto f^{2}\right)$ on the excitation amplitude have dramatic qualitative and quantitative effects on the force-response curves. 


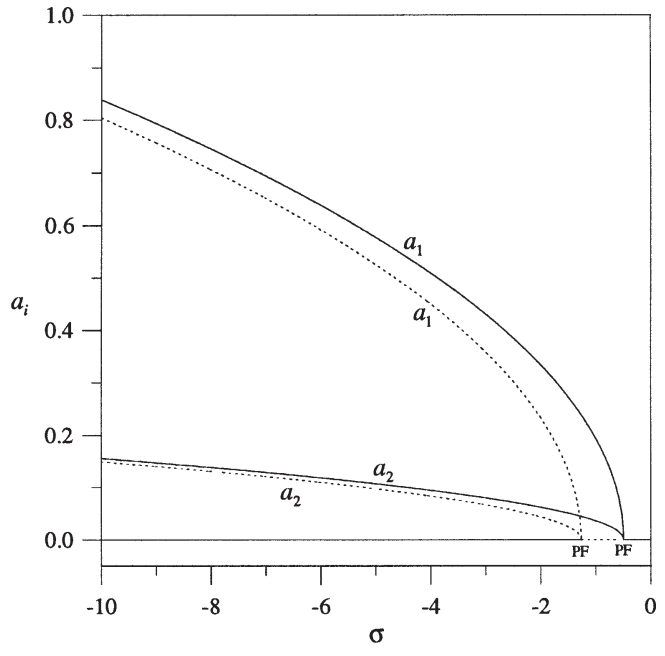

Fig. 7. Frequency-response curves for an additive-type external subcombination resonance involving modes 1 and 2 for $f=20$, $\mu_{1}=0.0137$ and $\mu_{2}=0.0635$. Solid lines $(-)$ denote stable fixed points and dashed lines $(---)$ denote unstable fixed points.

In Fig. 7, we show typical frequency-response curves for the same resonance when $f=20$. As in the case of combination parametric resonance, the curves are bent to the left, indicating a softening-type nonlinearity. Because $\Gamma_{1}$ and $\Gamma_{2}$ are negative and proportional to $f^{2}$, there is a strong decrease in the linear natural frequencies with an increase in $f$. Consequently, for $f=20$, unlike the combination parametric resonance, the external subcombination resonance is activated only for negative values of $\sigma$. We also note that increasing the forcing amplitude causes both the stable and unstable branches to shift to the left, with the latter being shifted more than the former.

In Fig. 8, we show typical amplitude-response curves for a subcombination external resonance of the first and third modes for $\sigma=-1$. Comparing Figs 6 and 8 , we note that the amplitude-response curves for the external subcombination resonance of modes 1 and 3 are qualitatively different from the amplitude-response curves for the external subcombination resonance of modes 1 and 2. As in Fig. 6, the trivial solution in Fig. 8 loses stability via a subcritical pitchfork bifurcation as $f$ is increased, resulting in a jump in the response amplitudes. However, the amplitudes of the nontrivial solutions in Fig. 8 increase as $f$ is increased, in contrast to the results in Fig. 6, where the amplitudes of the nontrivial solutions decrease as $f$ is increased.

Comparing Figs 3(a) and 5(a) with Fig. 8, we note that the amplitude-response curves for the external subcombination resonance of modes 1 and 3 are similar to those obtained for the combination parametric

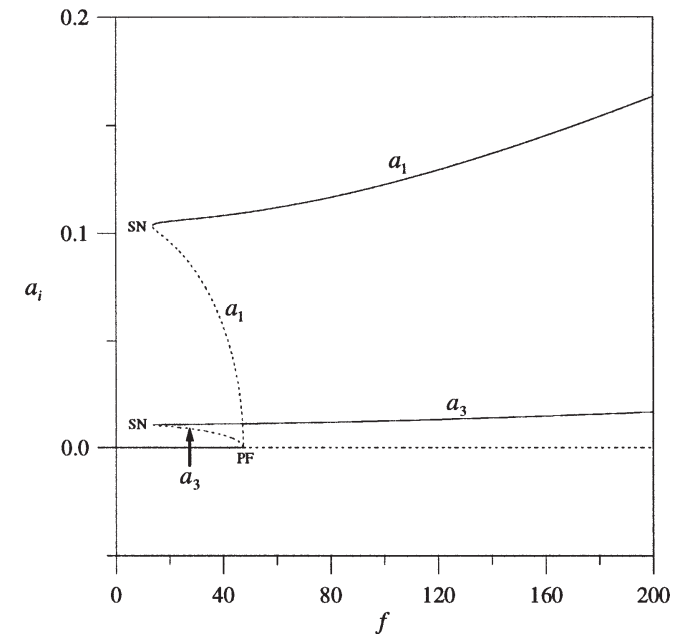

Fig. 8. Amplitude-response curves for an additive-type external subcombination resonance involving modes 1 and 3 for $\sigma=-1$, $\mu_{1}=0.0137$ and $\mu_{3}=0.076$. Solid lines $(-)$ denote stable fixed points and dashed lines (-- ) denote unstable fixed points.

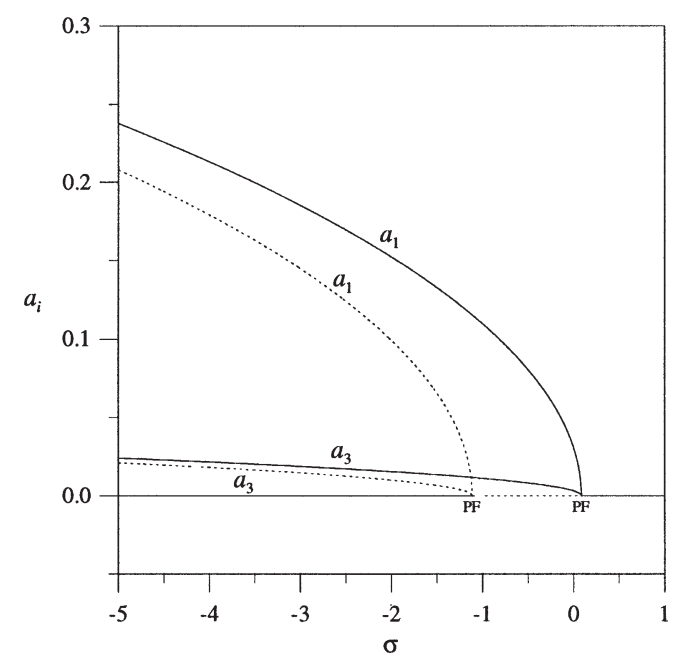

Fig. 9. Frequency-response curves for an additive-type external subcombination resonance involving modes 1 and 3 for $f=50$, $\mu_{1}=0.0137$ and $\mu_{3}=0.076$. Solid lines (-) denote stable fixed points and dashed lines (-- ) denote unstable fixed points.

resonance. Therefore, the effects of the linear shifts in the natural frequencies and the nonlinear dependence of the effective forcing $\Lambda$ on the excitation amplitude $f$ do not change qualitatively the amplitude-response curves.

In Fig. 9, we show typical frequency-response curves for the external subcombination resonance of modes 1 and 3 when $f=50$. Again the curves are bent to the left, indicating that the nonlinearity and the linear shift $\Gamma_{1} / 2 \omega_{1}$ decrease the frequency of the dominant first 
mode. Furthermore, similar to the combination parametric resonance, this case of external subcombination resonance may be activated for positive as well as negative values of $\sigma$.

Of the cases mentioned in Table 2, we found that the responses obtained for the external subcombination resonance of modes 2 and 4 are qualitatively similar to those obtained for the external subcombination resonance of modes 1 and 3, whereas the behaviors of the remaining cases are qualitatively similar to the external subcombination resonance of modes 1 and 2 .

Finally, we note again that the case of external subcombination resonance of the difference type can be studied by replacing $\omega_{m}$ by $-\omega_{m}$ and $\beta_{m}$ by $-\beta_{m}$ in Eqs (52)-(56). However, it can be seen from Eq. (64) that this resonance cannot be activated.

\section{Conclusion}

The nonlinear flexural responses of cantilever beams to combination parametric and subcombination resonances have been investigated. For the case of combination parametric resonance, the beam is excited longitudinally, whereas for the case of external subcombination resonance, the beam is excited transversely. In the parametric case, the Lagrangian and virtual-work term are discretized using a two-mode Galerkin technique and Hamilton's extended principle is used to obtain two second-order nonlinear ordinary-differential equations of motion. Then, the method of multiple scales is used to obtain a set of four first-order nonlinear ordinary-differential equations governing the modulation of the amplitudes and phases of the two excited modes. In the subcombination case, the method of time-averaged Lagrangian and virtual work along with Hamilton's extended principle are used to obtain the modulation equations.

We found that the excitation amplitude must exceed a certain threshold for either resonance to be activated. For the external subcombination resonance, two qualitatively different amplitude-response behaviors were found. In the first, the external subcombination resonance will not be activated if the excitation amplitude is chosen beyond a certain limit. In the second, similar to the case of combination parametric resonance, no upper limit on the excitation amplitude exists for the resonance to be activated.

In both parametric combination and external subcombination resonances, the trivial solution loses stability via pitchfork bifurcations, both supercritical and subcritical, thereby producing nontrivial responses. When the pitchfork bifurcation is supercritical, the change in amplitudes is gradual and therefore the transition is smooth. When the pitchfork bifurcation is subcritical, the change in amplitudes is abrupt and is associated with a jump. In addition, the nontrivial solutions lose stability via saddle-node bifurcations as the excitation amplitude is decreased below a critical value, resulting in a jump down to the trivial solution.

For cantilever beams, we found that combination parametric and external subcombination resonances of the difference type cannot be activated. Rather, only additive-type resonances can be excited.

\section{Acknowledgment}

This work was supported by the National Science Foundation under Grant No. CMS-9423774.

\section{Appendix A}

$$
\begin{aligned}
& \delta_{1}=\int_{0}^{1}\left(\int_{0}^{s} \phi_{m}^{\prime 2} \mathrm{~d} s\right)^{2} \mathrm{~d} s \\
& \delta_{2}=\delta_{7}=\int_{0}^{1}\left(\int_{0}^{s} \phi_{m}^{\prime 2} \mathrm{~d} s\right)\left(\int_{0}^{s} \phi_{m}^{\prime} \phi_{n}^{\prime} \mathrm{d} s\right) \mathrm{d} s \\
& \delta_{3}=\delta_{4}=\int_{0}^{1}\left(\int_{0}^{s} \phi_{m}^{\prime} \phi_{n}^{\prime} \mathrm{d} s\right)^{2} \mathrm{~d} s \\
& \delta_{5}=\delta_{9}=\int_{0}^{1}\left(\int_{0}^{s} \phi_{m}^{\prime} \phi_{n}^{\prime} \mathrm{d} s\right)\left(\int_{0}^{s} \phi_{n}^{\prime 2} \mathrm{~d} s\right) \mathrm{d} s \\
& \delta_{6}=\int_{0}^{1}\left(\int_{0}^{s} \phi_{n}^{\prime 2} \mathrm{~d} s\right)^{2} \mathrm{~d} s, \\
& \delta_{8}=\delta_{3}+\int_{0}^{1}\left(\int_{0}^{s} \phi_{m}^{\prime 2} \mathrm{~d} s\right)\left(\int_{0}^{s} \phi_{n}^{\prime 2} \mathrm{~d} s\right) \mathrm{d} s \\
& \alpha_{1}=\frac{1}{2} \int_{0}^{1} \phi_{m}^{\prime 2} \phi_{m}^{\prime \prime 2} \mathrm{~d} s, \\
& \alpha_{3}=\frac{1}{2} \int_{0}^{1}\left(\phi_{m}^{\prime \prime 2} \phi_{n}^{\prime 2}+4 \phi_{m}^{\prime} \phi_{m}^{\prime \prime} \phi_{n}^{\prime} \phi_{n}^{\prime \prime}+\phi_{m}^{\prime 2} \phi_{n}^{\prime \prime 2}\right) \mathrm{d} s,
\end{aligned}
$$




$$
\begin{aligned}
\alpha_{4} & =\int_{0}^{1}\left(\phi_{m}^{\prime} \phi_{n}^{\prime} \phi_{n}^{\prime \prime 2}+\phi_{m}^{\prime \prime} \phi_{n}^{\prime 2} \phi_{n}^{\prime \prime}\right) \mathrm{d} s, \\
\alpha_{5} & =\frac{1}{2} \int_{0}^{1} \phi_{n}^{\prime 2} \phi_{n}^{\prime \prime 2} \mathrm{~d} s, \\
\mu_{i} & =\frac{1}{2} \int_{0}^{1} c \phi_{i}^{2} \mathrm{~d} s, \\
f_{i j} & =\int_{0}^{1}\left[\phi_{i}^{\prime \prime}(s-1)+\phi_{i}^{\prime}\right] \phi_{j} f \mathrm{~d} s, \quad i, j=m, n .
\end{aligned}
$$

\section{Appendix B}

$$
\begin{aligned}
\Gamma_{i}= & \int_{0}^{1}\left[2 \phi_{i}^{\prime \prime 2} \Phi^{\prime 2}+8 \phi_{i}^{\prime} \phi_{i}^{\prime \prime} \Phi^{\prime} \Phi^{\prime \prime}\right. \\
& \left.+2 \phi_{i}^{\prime 2} \Phi^{\prime \prime 2}-2\left(\omega_{i}^{2}+\Omega^{2}\right)\left(\int_{0}^{s} \phi_{i}^{\prime} \Phi^{\prime} \mathrm{d} s\right)^{2}\right] \mathrm{d} s \\
\Lambda= & \int_{0}^{1}\left[2 \phi_{m}^{\prime} \phi_{n}^{\prime \prime} \Phi^{\prime} \Phi^{\prime \prime}+2 \phi_{m}^{\prime \prime} \phi_{n}^{\prime} \Phi^{\prime} \Phi^{\prime \prime}\right. \\
& +\phi_{m}^{\prime} \phi_{n}^{\prime} \Phi^{\prime \prime 2}+\phi_{m}^{\prime \prime} \phi_{n}^{\prime \prime} \Phi^{\prime 2} \\
& +\left(\Omega^{2}+\omega_{m} \omega_{n}-\omega_{m} \Omega-\omega_{n} \Omega\right) \\
& \times\left(\int_{0}^{s} \phi_{m}^{\prime} \Phi^{\prime} \mathrm{d} s\right)\left(\int_{0}^{s} \phi_{n}^{\prime} \Phi^{\prime} \mathrm{d} s\right) \\
& -\Omega\left(\omega_{m}+\omega_{n}\right) \\
& \left.\times\left(\int_{0}^{s} \phi_{m}^{\prime} \phi_{n}^{\prime} \mathrm{d} s\right)\left(\int_{0}^{s} \Phi^{\prime 2} \mathrm{~d} s\right)\right] \mathrm{d} s .
\end{aligned}
$$

\section{References}

[1] T.J. Anderson, B. Balachandran and A.H. Nayfeh, Nonlinear resonances in a flexible cantilever beam, J. Vibration and Acoustics, Trans. ASME 116 (1994), 480-484.
[2] K.G. Asmis and W.K. Tso, Combination and internal resonance in a nonlinear two-degrees-of-freedom system, J. Applied Mechanics, Trans. ASME 39 (1972), 832-834.

[3] M.P. Cartmell and J.W. Roberts, Simultaneous combination resonances in a parametrically excited cantilever beam, Strain 23 (1987), 117-126.

[4] J. Dugundji and V. Mukhopadhyay, Lateral bending-torsion vibrations of a thin beam under parametric excitation, J. Applied Mechanics, Trans. ASME 40 (1973), 693-698.

[5] R.C. Kar and T. Sujata, Parametric instability of an elastically restrained cantilever beam, Computers and Structures 34 (1990), 469-475.

[6] R.C. Kar and T. Sujata, Dynamic stability of a rotating, pretwisted and preconed cantilever beam including coriolis effects, Computers \& Structures 42 (1992), 741-750.

[7] A.H. Nayfeh, Introduction to Perturbation Techniques, WileyInterscience, New York, 1981.

[8] A.H. Nayfeh, Combination resonances in the non-linear response of bowed structures to a harmonic excitation, J. Sound and Vibration 90 (1983), 457-470.

[9] A.H. Nayfeh and D.T. Mook, Nonlinear Oscillations, WileyInterscience, New York, 1979.

[10] A.H. Nayfeh and L.D. Zavodney, The response of two-degreeof-freedom systems with quadratic non-linearities to a combination parametric resonance, J. Sound and Vibration 107 (1986), 329-350.

[11] J. Shaw, S.W. Shaw and A.G. Haddow, On the response of the non-linear vibration absorber, Int. J. Non-Linear Mechanics 24 (1989), 281-293.

[12] S. Sridhar, A.H. Nayfeh and D.T. Mook, Nonlinear resonances in a class of multi-degree-of-freedom systems, J. Acoustical Society of America 58 (1975), 113-123.

[13] D.A. Streit, A.K. Bajaj and C.M. Krousgrill, Combination parametric resonance leading to periodic and chaotic response in two-degree-of-freedom systems with quadratic nonlinearities, J. Sound and Vibration 124 (1988), 297-314.

[14] S. Timoshenko, Vibration Problems in Engineering, Van Nostrand, New York, 1928.

[15] W.K. Tso and K.G. Asmis, Multiple parametric resonance in a non-linear two degree of freedom system, Int. J. Non-Linear Mechanics 9 (1974), 269-277.

[16] T. Yamamoto, K. Yasuda and N. Tei, Summed and differential harmonic oscillations in a slender beam, Bulletin JSME 24 (1981), 1214-1222.

[17] T. Yamamoto, K. Yasuda and N. Tei, Super summed and differential harmonic oscillations in a slender beam, Bulletin JSME 25 (1982), 959-968. 

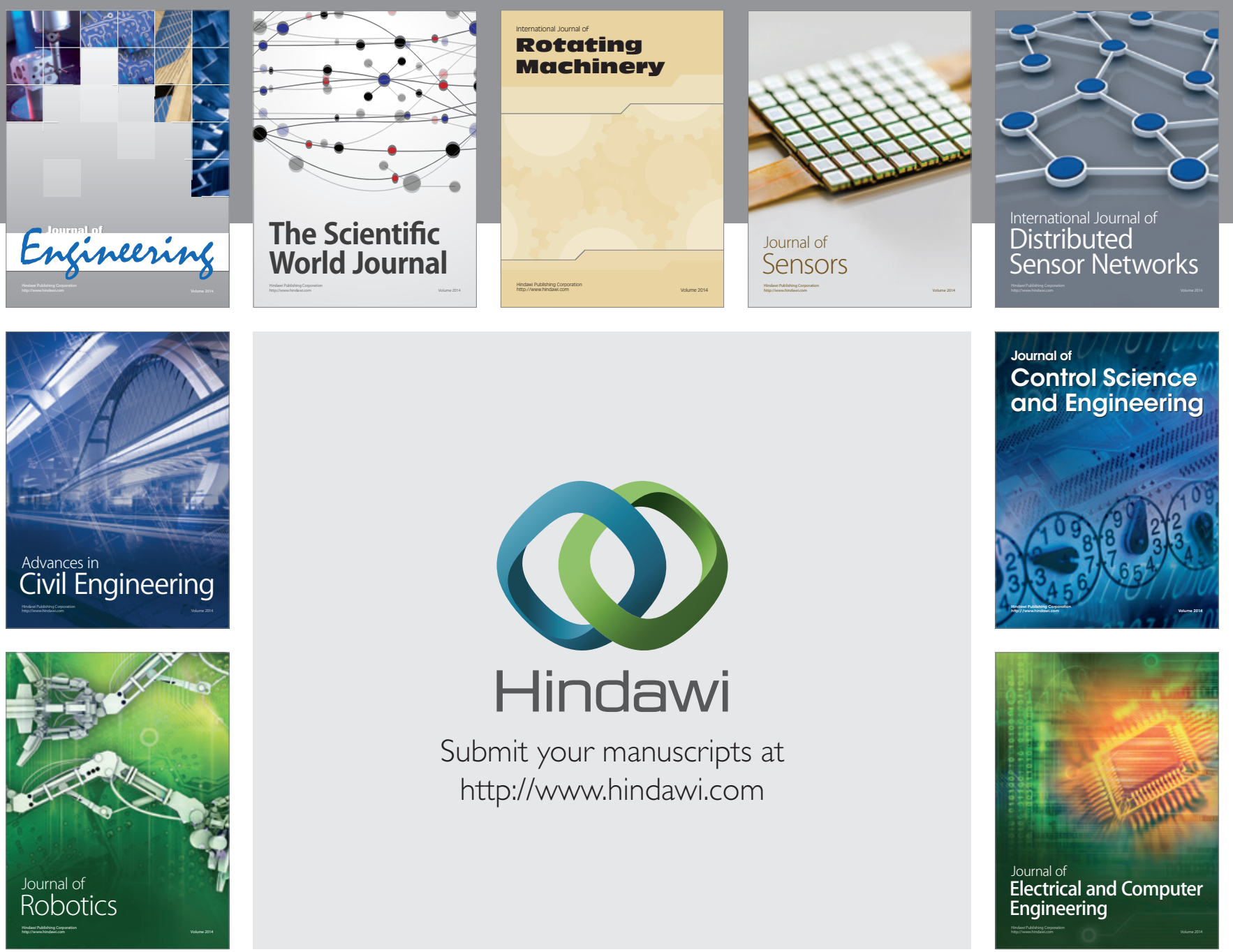

Submit your manuscripts at

http://www.hindawi.com
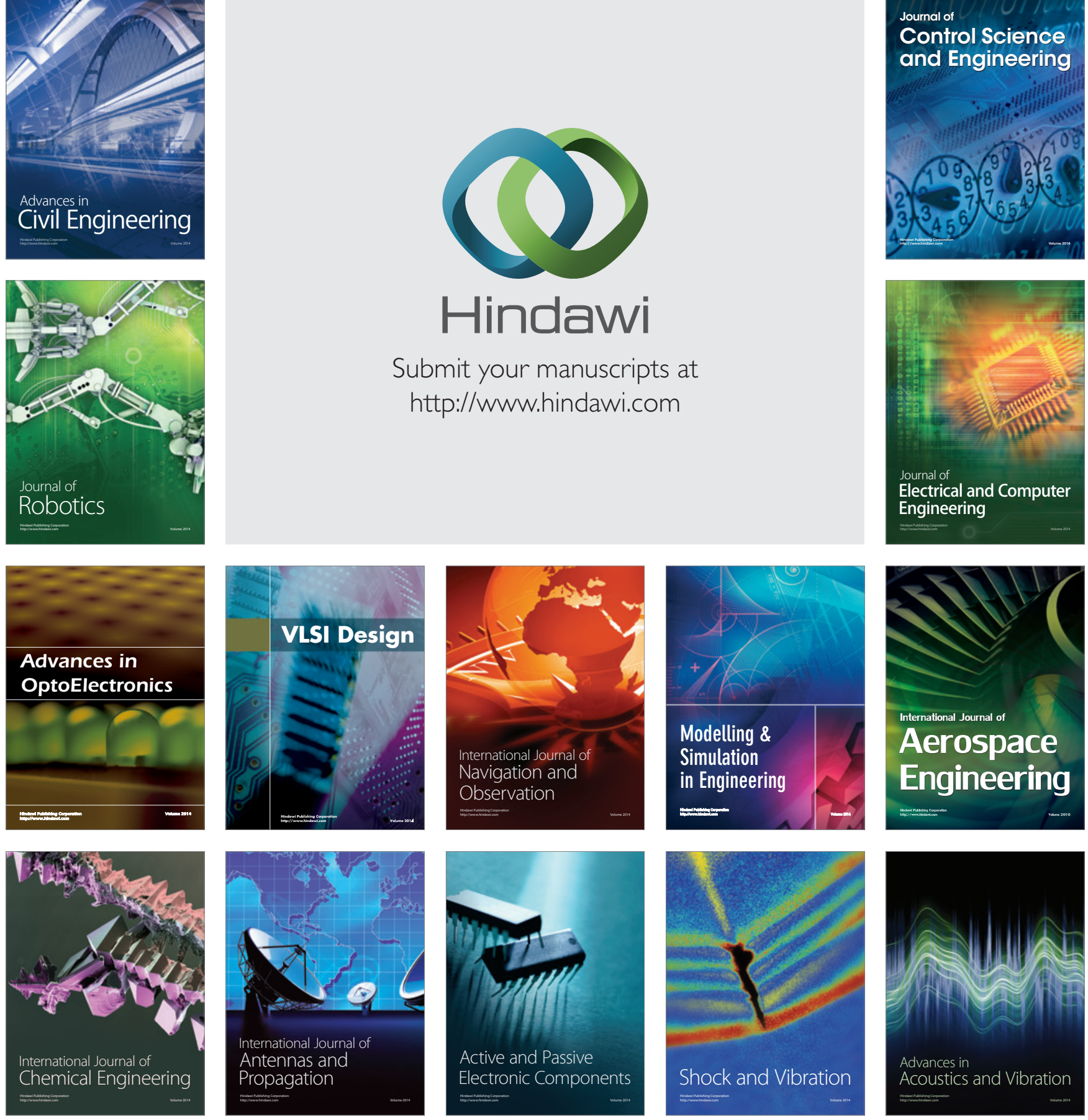